\title{
BMJ Open Effects of a 12-week cardiovascular rehabilitation programme on systemic inflammation and traditional coronary artery disease risk factors in patients with rheumatoid arthritis (CARDIA trial): a randomised controlled trial
}

\author{
Stefan Heinze-Milne, ${ }^{1}$ Volodko Bakowsky, ${ }^{2}$ Nicholas Giacomantonio, ${ }^{3}$ \\ Scott A Grandy ${ }^{1,3,4}$
}

To cite: Heinze-Milne $S$, Bakowsky V, Giacomantonio N, et al. Effects of a 12-week cardiovascular rehabilitation programme on systemic inflammation and traditional coronary artery disease risk factors in patients with rheumatoid arthritis (CARDIA trial): a randomised controlled trial. BMJ Open 2017;7:e018540. doi:10.1136/ bmjopen-2017-018540

\section{- Prepublication history for} this paper is available online. To view these files, please visit the journal online (http://dx.doi. org/10.1136/bmjopen-2017018540).

Received 6 July 2017

Revised 20 November 2017

Accepted 23 November 2017

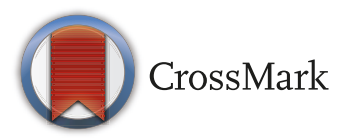

${ }^{1}$ School of Health and Human Performance, Dalhousie University, Halifax, Canada ${ }^{2}$ Division of Rheumatology, Department of Medicine, Nova Scotia Health Authority, Halifax, Canada

${ }^{3}$ Division of Cardiology, Nova Scotia Health Authority, Halifax, Canada

${ }^{4}$ Department of Pharmacology, Dalhousie University, Halifax, Canada

Correspondence to Dr Scott A Grandy; grandy@dal.ca

\section{ABSTRACT}

Introduction Patients with systemic inflammatory diseases such as rheumatoid arthritis (RA) have an increased risk of cardiovascular disease (CVD) above the baseline risk attributable to traditional CVD risk factors seen in the general population. Exercise in cardiac rehabilitation (CR) is designed specifically for high-risk primary prevention and those with established CVD. Even though the European League Against Rheumatism guidelines state that exercise is safe for individuals with RA and exercise can reduce CVD risk, patients with RA rarely participate in CR. Thus, little is known about CR's impact on inflammatory and CVD risk in the RA population. The purpose of this trial is to determine the feasibility of a 12-week CR programme for patients with RA and whether it decreases CVD risk without exacerbating RA.

Methods and analysis This is a randomised controlled trial whereby 60 participants with RA will be recruited and randomly assigned to either standard of care (SOC) treatment or SOC plus a 12-week CR programme $(60 \mathrm{~min}$ of education plus two $60 \mathrm{~min}$ aerobic exercise sessions/ week). Exercise will be performed at $60 \%-80 \%$ of heart rate reserve. Outcome measures (Framingham Risk Score, resting heart rate, blood pressure, blood lipids, markers of systemic inflammation (ie, interleukin (IL) 6 and tumour necrosis factor- $\alpha$ (TNF- $\alpha$ ), Clinical Disease Assessment Index, Disease Activity Score-28, physical activity levels and peak cardiorespiratory fitness) will be assessed preintervention (week-0), postintervention (week-13) and 6 months postintervention.

Ethics and dissemination Ethical approval was obtained from the Nova Scotia Health Authority Research Ethics Board. Results will be submitted for publication in an appropriate peer-reviewed journal.

Trial registration number NCT01534871; Pre-results

\section{INTRODUCTION}

Rheumatoid arthritis (RA) is a chronic inflammatory autoimmune disease that combines joint tenderness and swelling and synovial

\section{Strengths and limitations of this study}

- The determination of the impact of exercise on the primary cardiovascular disease (CVD) risk factor (inflammation) in individuals with rheumatoid arthritis (RA).

- The determination of whether cardiac rehabilitation can be used to reduce CVD risk without exacerbating RA.

- The objectives of the study are determined using a robust randomised controlled methodology and a repeated measures design, including a 6-month follow-up.

- The study is limited in that any potential participants taking a statin were excluded from the study. The high use of statins in primary prevention of CVD has likely prevented a significant number of individuals, who may have benefited from the intervention, from participating.

- The study is limited in that only the impact of vigorous aerobic activity $(60 \%-80 \%$ of heart rate reserve) will be examined.

joint degradation. ${ }^{1}$ Individuals with RA are at increased risk for mortality compared with the general population, ${ }^{23}$ particularly due to their increased risk of cardiovascular disease (CVD). ${ }^{4}$ Accelerated coronary artery atherosclerosis is believed to be directly responsible for the increase in CVD-related events and deaths. ${ }^{356}$ Although traditional risk factors contribute to the development of CVD in individuals with $\mathrm{RA}$, as well as in the general population, they do not fully explain the higher incidence of CVD events observed in the RA population..$^{7-9}$ A primary contributing factor for increased CVD risk and cardiovascular (CV) events in individuals with RA is likely the elevated levels of systemic inflammation which are part of 
the RA condition. ${ }^{10}$ Specifically, the inflammation associated with RA increases CVD risk via its impact on vessel wall health, as well as through the adverse modulation of traditional CVD risk factors. ${ }^{11} 12$ Further, patients with RA on average have residually higher CVD risk due to having lower cardiorespiratory fitness (CRF) levels as compared with their non-RA counterparts. ${ }^{13}$ Therefore, therapeutic interventions that target both traditional risk factors and systemic inflammation are needed to reduce CVD in the RA population.

One potential intervention that could be used to decrease CVD in the RA population is exercise. It is accepted that regular vigorous-intensity exercise decreases CVD risk factors in both healthy and chronic disease populations. ${ }^{14}$ Exercise also increases CRF, which is inversely related with levels of systemic inflammation ${ }^{15-17}$ and CVD mortality. ${ }^{18}$ As such, this approach may reduce CVD risk and inflammation in patients with RA, as it is well established to decrease risk/events in both primary and secondary prevention of CVD populations. ${ }^{19}{ }^{20}$ For a large proportion of individuals, this exercise prescription is administered as part of a cardiac rehabilitation (CR) programme. There is considerable evidence that shows CR programme decreases CVD risk and events. ${ }^{21}$

Current European League Against Rheumatism (EULAR) recommendations also advise that exercise can be used to help manage levels of inflammation with RA (via $\mathrm{C}$ reactive protein (CRP) reduction). ${ }^{22}$ Despite this information and their increased risk of CVD, patients with RA are not typically referred to or attend CR. ${ }^{2324}$ This is in part because there is still some belief among patients that exercise could exacerbate RA, ${ }^{25}$ despite studies showing exercise to be safe. ${ }^{26-29}$ In order to improve the rationale and get more patients with RA active, more knowledge of how exercise affects those with RA is needed. Recent trials have demonstrated that structured exercise can decrease cardiovascular risk, CRP levels and rheumatoid disease activity in individuals with RA. ${ }^{30-32}$ However, very little research has been conducted regarding the influence of exercise on other inflammatory cytokines known to be involved in both RA and atherosclerotic development (ie, interleukin 6, TNF- $\alpha$, etc). Thus, the full impact of exercise on CVD risk and systemic inflammation associated with RA is not yet well characterised.

Although the EULAR guidelines recommend general exercise, there are currently no structured exercise guidelines for patients with RA aimed at reducing CVD risk or levels of systemic inflammation. The CR model is a good starting point to determine the effects of a common standardised exercise programme on CVD risk and systemic inflammation in patients with RA. Thus, the purpose of this study is to determine the feasibility and effects of a standardised 12-week CR programme (vigorous aerobic exercise and education) on traditional CVD risk and systemic inflammation in individuals with RA. The specific study objectives are: (1) to determine the effects of a CR programme on traditional CVD risk factors (blood lipids, hypertension, etc) and systemic markers of inflammation in patients with RA, (2) to determine the effects of a CR programme on CVD risk in patients with RA, (3) to determine whether a CR programme influences the severity of RA and/or improves patient's functional ability and (4) to examine the relationship between RA severity, CVD risk and systemic inflammation. The primary outcome measure is the Framingham CVD Risk Score (FRS). Secondary outcome measures include levels of systemic inflammation as measured in the serum, resting heart rate (RHR), blood pressure (BP), blood lipids, the American College of Rheumatology (ACR) response score, the Clinical Disease Assessment Index (CDAI), the Disease Activity Score (DAS28), physical activity (PA) levels and CRF. It is hypothesised that CR will decrease traditional risk factors and systemic inflammation, and thus decrease CVD risk in those with RA. It is expected that RA severity will not change with exercise prescription.

\section{METHODS}

\section{Study design}

This study is a randomised controlled trial with repeated measures. Participants will be randomly assigned to either (1) standard of care (SOC) treatment $(\mathrm{CON})$ or (2) SOC plus a 12-week CR programme (EX). Outcome measures will be assessed at baseline (week 0), postintervention (week 12) and at a 6-month follow-up. The primary outcome measure is the FRS. Secondary outcome measures include levels of systemic inflammation (eg, serum levels of IL-6, IL-10, TNF- $\alpha$, etc), severity of RA, weight, waist girth, RHR, resting BP, lipid profile, fasting glucose, aerobic fitness, PA levels, functional ability, tobacco use and alcohol consumption.

\section{Sample}

All participants will be recruited from an outpatient rheumatology clinic at the Nova Scotia Rehabilitation Centre (NSHA Central Zone, Halifax, Nova Scotia). Individuals with RA will be screened by their rheumatologist during their clinic visit based on the inclusion/exclusion criteria described below. If the patient meets the eligibility criteria, they will be asked if they are interested in participating in a research study. Those patients who are interested in participating will be contacted by the trial coordinator who will explain the purpose and requirements of the study and complete the informed consent with those individuals who wish to enter the trial.

\section{Sample size}

Sample size for the study was calculated using the primary outcome measure of interest, CVD risk as determined using the FRS. Previous work has shown that 16 weeks of aerobic exercise resulted in a significant difference in FRS score (-2.25 points) ${ }^{33}$ Thus, a 2.25-point improvement in the FRS was used to calculate sample size. Based on this difference and the ability to achieve $90 \%$ power $(\alpha=0.05)$ the current study requires 25 participants per group. An attrition rate of $20 \%$ is assumed for the study, therefore 
30 participants/group will be recruited to ensure that 25 participants/group complete the study.

\section{Inclusion/exclusion criteria}

Only those patients who (1) are 20 years of age or older, (2) have RA fulfilling the ACR criteria, ${ }^{1}$ (3) are receiving stable pharmacotherapy (defined as $\geq 3$ months of unchanged antirheumatic drugs and $\geq 1$ month of unchanged non-steroidal anti-inflammatory drugs), (4) have moderate or high risk of CVD, but not receiving a statin, (5) are able to walk on treadmill or cycle on a stationary bike for $15 \mathrm{~min}$ and (6) are able to attend the CR programme (held in the Halifax Regional Municipality) will be invited to participate in the study. Patients will be excluded from the study if they are (1) diabetic; (2) have known coronary, cerebral or peripheral artery disease; (3) taking statins; (4) have one or more arthroplasties of weight bearing joints or (5) are performing regular exercise (as defined by scoring in the 'high' category of the International Physical Activity Questionnaire (IPAQ).$^{34}$ Of note, CVD risk was determined based on age and the presence of CVD risk factors (smoking, hypertension, obesity, diabetes, etc). For the purpose of this study, males 45 years of age or older or males younger than 45 years with two or more CVD risk factors are considered to be at moderate risk of CVD. ${ }^{35}$ Females 55 years of age or older or females younger than 55 years with two or more CVD risk factors are considered to be at moderate risk of CVD. ${ }^{35}$ Only individuals who have signs or symptoms of CVD or have been diagnosed with CVD are considered at high risk. High-risk CVD individuals are only eligible to participate in the study if they have not been prescribed a statin for whatever reason. If an individual is prescribed a statin during the 12-week intervention, they will be withdrawn from the study. Of note, since there is a high prevalence of depression in patients with $\mathrm{RA}^{36}$ and depression may influence inflammation, ${ }^{37}$ any diagnosis of depression will be noted in the participant file so that the participants can be identified during analysis.

\section{Patient screening}

If the individual agrees to participate in the study, they will be asked about their alcohol and tobacco use, have their BP measured and sent for routine blood work. Blood tests will determine the patient's lipid profile (total cholesterol, low-density lipoprotein cholesterol, high-density lipoprotein cholesterol and triglycerides) and CRP. Patient history and blood test results will be used to determine the patient's FRS ${ }^{38}$ which estimates the 10 -year CV risk of the patient. FRS scores will be adjusted based on the participant's CRP level.

\section{Participant group assignment}

The group allocation sequence was generated using a computer-based random number generator (Research Randomizer, www.randomizer.org), which generated a set of 60 non-unique numbers ( $1=$ exercise group and 2=controlgroup). Physicians conducting the RA/CVD assessments are blinded to participant group assignment.
Only the principle investigator, research coordinator and the CR staff will know which group the participant has been assigned to. Participants are only assigned to an intervention group once they have been consented.

\section{Outcome measures}

RA and CVD outcomes as well as PA will be measured in all participants (CON and EX) at three different time points during the study. Participants will be assessed at baseline (week 0 ) and reassessed postintervention (12weeks) as well as 6 months after the completion of the 12-week intervention. The postintervention assessment will be conducted within 48-72hours after the completion of the final exercise session. Participants will be contacted 2 weeks prior to their 6-month anniversary of the completion of the CR programme to schedule their 6-month follow-up assessment. This will allow the assessments to be conducted within 2 weeks of their anniversary date. The techniques that will be used to assess these outcomes are described below.

\section{CVD risk}

It will be assessed using the FRS as described in the 2012 Update of the Canadian Cardiovascular Society guidelines for the diagnosis and treatment of dyslipidemia for the prevention of cardiovascular disease in the adult. ${ }^{39}$ Since inflammation confers significant risk for the development of CVD in patients with RA, the participants FRS will be adjusted based on the serum levels of the biomarker high-sensitivity CRP. ${ }^{40}$

\section{RA severity}

The ACR response score will be used to assess RA severity. ${ }^{41}$ The ACR is a composite score based on seven measures: three by an assessor (swollen joint, tender joint count and physician global status), three by the patient (function, pain and global status) and one acute phase measure (CRP and erythrocyte sedimentation rate). The ACR examination will be conducted by a rheumatologist. The DAS-28 and CDAI tools are also used to measure RA severity.

\section{Peak aerobic fitness}

It will be predicted based on the results from a stress test. These tests will be performed at the Queen Elizabeth II Health Sciences Centre (Halifax, Nova Scotia) and are considered SOC for entry into the CR programme. Participants will perform a graded exercise test. ${ }^{42}$ The typical treadmill protocols used include the Bruce or modified Bruce treadmill tests. ${ }^{42}{ }^{43}$ For each test BP and a 12-lead ECG will be monitored to determine if there is any evidence of CVD. The test will be terminated based on the American College of Cardiology / the American Heart Association's (ACC/ AHA) 2002 guidelines for exercise testing. ${ }^{44}$ All tests will be conducted by a certified stress test technician and overseen by a cardiologist. Predictive equations will be used to calculate the participant's peak oxygen uptake. ${ }^{45} 46$

\section{Physical activity}

This will be quantified using the long form of the IPAQ. ${ }^{47}$ The IPAQ has been shown to be a reliable and valid method to assess $\mathrm{PA}^{47}$ In addition, the IPAQ has been used in 
numerous studies to assess PA as well as in RA studies with smaller sample sizes. ${ }^{48-50}$

\section{Functional ability}

Functional status will be assessed using the short form of the Health Assessment Questionnaire (HAQ) ${ }^{5152}$ This self-report questionnaire is composed of the HAQ disability index, HAQ patient global health status an $\mathrm{d}$ the visual analogue scale for pain. ${ }^{52} 53$

\section{Blood analysis}

Venous blood samples will be drawn according to standard operating procedure by the research coordinator (registered nurse). Samples will be processed at the local hospital to determine the participant's lipid profile, fasting glucose and CRP levels. Serum also will be extracted from the blood samples in order to quantify cytokine levels (IL-1 $\alpha$, IL-1 $\beta$, IL-4, IL-6, IL-10, IL-17 and TNF- $\alpha$ ), which are an indicator of systemic inflammation. Human multiplex cytokine assays (Bio-Rad Laboratories, Mississauga, Ontario) will be prepared in accordance to the manufacturer's instructions to determine the cytokine levels in the serum. A Bio-Plex suspension array system will be used to analyse the cytokine assay. All serum samples will be stored at $-20^{\circ} \mathrm{C}$ until they are required for analysis.

\section{Feasibility and patient safety}

The feasibility of the programme will be measured in terms of patient retention, the impact on the patient's RA (eg, ACR response score) and the patient's perceived benefit of the programme. To assess retention participants will be given an exercise log book and asked to record the number of exercise and education sessions that they attend each week. Patients also will be asked to complete the standard CR programme feedback questionnaire to determine how effective they perceived the programme to be. The questionnaire will evaluate both the education and exercise components of the CR programme. To monitor safety, participants will be instructed to report and record any adverse events that are associated with the CR programme. As part of the orientation to the CR programme, the participants will be instructed as to what constitutes an adverse event (eg, cardiovascular, RA, musculoskeletal, etc).

\section{Exercise intervention}

Participants assigned to the $\mathrm{CON}$ group will receive the treatment for their RA that is considered SOC (eg, pharmacotherapy), but will not be enrolled in the CR programme. These participants will be asked to maintain their current level of PA throughout the course of the study. Participants assigned to EX will receive SOC for RA plus be enrolled in a CR programme (Community Cardiovascular Hearts Motion programme, Halifax Regional Municipality, Nova Scotia). The CR programme is 12 weeks in duration (supervised by a nurse and a physiotherapist) and is composed of one $60 \mathrm{~min}$ education session and two $60 \mathrm{~min}$ exercise sessions per week. Education sessions will be conducted by a nurse, physiotherapist or a dietician and cover topics such as heart healthy eating, setting health-related goals, exercise, nutrition, healthy weight, smoking cessation and stress/coping. The education sessions are specific to those with or at risk of developing CVD. None of the education sessions will deal specifically with RA, but all of the topics discussed will be applicable to the RA population. Thus, it is expected the education sessions will be highly attended by the study participants. The exercise sessions are based on the SOC for patients with CVD. The number of exercise sessions per week (two) is the protocol used by the Community Cardiovascular Hearts in Motion programme. Participants will perform their aerobic exercise in a room that houses multiple pieces of aerobic exercise equipment. Participants will perform their exercise prescription on their own piece of aerobic equipment, thus the exercise is individualised. However, since the equipment is close enough together to allow participants to converse with one another, it will create a quasi-group setting. In the group, it is common for patients to be exercising at different intensities since levels of exercise tolerance will differ between participants. Sessions begin with a group warm-up activity, followed by $45 \mathrm{~min}$ of aerobic activity and finish with a cool down. The activities include walking/running on a treadmill, stepping exercise and/or cycling on a cycle ergometer (participant may rotate through the various exercise modalities). Participants will exercise at a vigorous intensity (heart rate reserve $60 \%-80 \%$ ). Prior to the initial CR session, target heart rates (HRs) will be calculated for each participant based on the maximum HR achieved during their stress test. Participants will be taught how to palpate radial and/or carotid pulse during the first week of the CR programme. This will enable the participant to monitor and maintain their HR within the target HR zone while performing their aerobic exercise. A nurse, physiotherapist and/or a research assistant will closely monitor exercise sessions. Participants will be given an exercise log to record RHR, type of exercise performed and duration of exercise and HR during each exercise session.

As stated above, it is expected that the exercise intensity (eg, target HRs) will vary between participants since the target HRs are based on the participant's stress test results. It is not anticipated that having study participants exercising at different intensities (target HRs) within the same CR session will lead to cross-contamination of the study exercise groups. To help ensure that cross-contamination does not occur, participants will be told that their exercise programme is based on the results of their stress test and specific to them that they should adhere to their individualised exercise prescription.

\section{Data analysis and management}

For each objective, the main outcome for the study will be the changes that occur between preintervention and postintervention (12weeks). Secondary analysis will be conducted to determine if the effects observed at the end of the 12-week intervention are maintained long term (6 months).

Objective 1: determine the effects of CR on CVD risk factors and systemic markers of inflammation in patients with $R A$. A factorial 
analysis of variance (ANOVA) with a Tukey's honestly significant difference (HSD) post hoc test will test for differences between CON and EX groups in CVD risk factors and markers of systemic inflammation between baseline, 12 weeks and the 6-month follow-up.

Objective 2: determine the effects of $C R$ and exercise intensity on CVD risk in patients with $R A$. A factorial ANOVA with a Tukey's HSD post hoc test will test for differences between CON and EX groups in markers of cardiac risk between baseline, 12 weeks, and the 6-month follow-up.

Objective 3: determine whether CR has a therapeutic effect on severity of $R A$ and improves functional ability in patients with $R A$. A factorial ANOVA with a Tukey's HSD post hoc test will test for differences between CON and EX groups in RA severity between baseline, 12 weeks and the 6-month follow-up.

Objective 4: to determine whether it is feasible and safe for patients with RA to participate in a CR programme. Adherence will be calculated as the number of sessions attended divided by the total number of sessions in the 12-week programme for each patient. Adherence will be calculated separately for the exercise and education components of the programme. Safety will be determined by examining the total number of adverse events that occur over the dura tion of the 12-week exercise programme. The total number of adverse events over the course of the study will be divided by the total number of participant hours to determine the number of adverse events per participant hour. Descriptive statistics will be used to characterise adherence to the intervention and the safety.

Data management: all data management procedures may be found at ClinicalTrials.gov.

\section{Ethics and dissemination}

The knowledge translation plan includes the presentation of the results at a national and/or international conference and the publication of articles in peer-reviewed journals. The authors declare no competing interests. The principal investigator will have access to the final trial dataset.

\section{DISCUSSION}

Individuals with RA are at increased risk of CVD, in part due to increased chronic levels of systemic inflammation from their disease. ${ }^{10}$ This inflammation facilitates plaque progression in the vasculature. ${ }^{11}{ }^{12}$ Patients with RA typically have lower CRF which also contributes to an increased CVD risk. ${ }^{13}$ Thus, there is an immediate need to improve the CVD risk profile of patients with RA. One way to do this is through aerobic exercise, which is offered as part of CR programme. There is strong evidence that CR programme improves the inflammatory profile in patients with CVD, as well as increase aerobic fitness. ${ }^{15-17}$ As such, it is reasonable to expect that the patients with RA would also benefit from CR programme in terms of decreasing their CVD risk. However, the current SOC for patients with RA does not typically involve referral to a CR programme ${ }^{2324}$ even though aerobic activity has been shown to be safe and effective for patients with RA. ${ }^{29}$
The importance of combining a current RA SOC programme and an aerobic exercise intervention could prove to be synergistic in the management of RA, and thus is important to investigate. However, currently, sufficient information is not there regarding its efficacy to make an informed decision to incorporate it into routine care. Available studies describe how aerobic exercise is safe and can provide improved functional ability for those with RA, ${ }^{30-32}$ yet our understanding of whether exercise reduces CVD risk and systemic inflammation in this population is lacking. The present study will contribute to our growing knowledge of this interaction and will help to create a better SOC model for patients with RA

Contributors SH-M has made substantial contributions to the acquisition, analysis and interpretation of the data, as well as drafting and critically revising the manuscript. SAG, NG and VB conceived and designed the trial and critically revised the manuscript. All authors have approved the final version of the manuscript and have agreed to be accountable for all aspects of the work.

Funding Funding for this study is provided by the Nova Scotia Health Research Foundation (Med-Research Programs-2012-8739).

Disclaimer The funder was not involved in the study design and is not involved with the implementation of study (eg, data collection, data analysis, interpretation and knowledge translation).

Competing interests None declared.

Patient consent Obtained.

Ethics approval Nova Scotia Health Authority Research Ethics Board (REB\#2012-355).

Provenance and peer review Not commissioned; externally peer reviewed.

Open Access This is an Open Access article distributed in accordance with the Creative Commons Attribution Non Commercial (CC BY-NC 4.0) license, which permits others to distribute, remix, adapt, build upon this work non-commercially, and license their derivative works on different terms, provided the original work is properly cited and the use is non-commercial. See: http://creativecommons.org/ licenses/by-nc/4.0/

(c) Article author(s) (or their employer(s) unless otherwise stated in the text of the article) 2017. All rights reserved. No commercial use is permitted unless otherwise expressly granted.

\section{REFERENCES}

1. Aletaha D, Neogi T, Silman AJ, et al. 2010 Rheumatoid arthritis classification criteria: an American College of Rheumatology/ European League Against Rheumatism collaborative initiative. Arthritis Rheum 2010;62:2569-81.

2. Sokka T, Abelson B, Pincus T. Mortality in rheumatoid arthritis: 2008 update. Clin Exp Rheumatol 2008;26:S35-61.

3. Kaplan MJ. Cardiovascular complications of rheumatoid arthritis: assessment, prevention, and treatment. Rheum Dis Clin North Am 2010;36:405-26.

4. Goodson N. Coronary artery disease and rheumatoid arthritis. Curr Opin Rheumatol 2002;14:115-20.

5. Chung CP, Oeser A, Raggi P, et al. Increased coronary-artery atherosclerosis in rheumatoid arthritis: relationship to disease duration and cardiovascular risk factors. Arthritis Rheum 2005;52:3045-53.

6. Erb N, Pace AV, Douglas KM, et al. Risk assessment for coronary heart disease in rheumatoid arthritis and osteoarthritis. Scand $J$ Rheumatol 2004;33:293-9.

7. del Rincón ID, Williams K, Stern MP, et al. High incidence of cardiovascular events in a rheumatoid arthritis cohort not explained by traditional cardiac risk factors. Arthritis Rheum 2001;44:2737-45.

8. Rho YH, Chung CP, Oeser A, et al. Inflammatory mediators and premature coronary atherosclerosis in rheumatoid arthritis. Arthritis Rheum 2009;61:1580-5.

9. Kozera L, Andrews J, Morgan AW. Cardiovascular risk and rheumatoid arthritis-the next step: differentiating true soluble 
biomarkers of cardiovascular risk from surrogate measures of inflammation. Rheumatology 2011;50:1944-54.

10. Sattar N, McCarey DW, Capell H, et al. Explaining how 'high-grade' systemic inflammation accelerates vascular risk in rheumatoid arthritis. Circulation 2003;108:2957-63.

11. Skeoch S, Bruce IN. Atherosclerosis in rheumatoid arthritis: is it all about inflammation? Nat Rev Rheumatol 2015;11:390-400.

12. Mahmoudi M, Aslani S, Fadaei R, et al. New insights to the mechanisms underlying atherosclerosis in rheumatoid arthritis. Int $J$ Rheum Dis 2017;20:287-97.

13. Metsios GS, Koutedakis $\mathrm{Y}$, Veldhuijzen van Zanten JJ, et al. Cardiorespiratory fitness levels and their association with cardiovascular profile in patients with rheumatoid arthritis: a crosssectional study. Rheumatology 2015;54:kev035-20.

14. Tremblay MS, Warburton DE, Janssen I, et al. New Canadian physical activity guidelines. Appl Physiol Nutr Metab 2011;36:36-46.

15. Handschin C, Spiegelman BM. The role of exercise and PGC1alpha in inflammation and chronic disease. Nature 2008;454:463-9.

16. Lavie CJ, Church TS, Milani RV, et al. Impact of physical activity, cardiorespiratory fitness, and exercise training on markers of inflammation. J Cardiopulm Rehabil Prev 2011;31:137-45.

17. Kim YJ, Shin YO, Bae JS, et al. Beneficial effects of cardiac rehabilitation and exercise after percutaneous coronary intervention on hsCRP and inflammatory cytokines in CAD patients. Pflugers Arch 2008;455:1081-8.

18. Kodama S, Saito K, Tanaka S, et al. Cardiorespiratory fitness as a quantitative predictor of all-cause mortality and cardiovascular events in healthy men and women: a meta-analysis. JAMA 2009;301:2024-35.

19. Piepoli MF, Corrà U, Adamopoulos $S$, et al. Secondary prevention in the clinical management of patients with cardiovascular diseases. Core components, standards and outcome measures for referral and delivery: A policy statement from the cardiac rehabilitation section of the european association for cardiovascular prevention $\&$ rehabilitation. Endorsed by the committee for practice guidelines of the european society of cardiology. Eur J Prev Cardiol 2014;21:664-81.

20. Redberg RF, Benjamin EJ, Bittner V, et al. AHA/ACCF 2009 performance measures for primary prevention of cardiovascular disease in adults. Circulation 2009:120:1296-336.

21. Taylor RS, Brown A, Ebrahim S, et al. Exercise-based rehabilitation for patients with coronary heart disease: systematic review and meta-analysis of randomized controlled trials. Am J Med 2004;116:682-92.

22. Agca R, Heslinga SC, Rollefstad S, et al. EULAR recommendations for cardiovascular disease risk management in patients with rheumatoid arthritis and other forms of inflammatory joint disorders: 2015/2016 update. Ann Rheum Dis 2017;76:17-28.

23. Metsios GS, Stavropoulos-Kalinoglou A, Veldhuijzen van Zanten $\mathrm{JJ}$, et al. Rheumatoid arthritis, cardiovascular disease and physical exercise: a systematic review. Rheumatology 2008;47:239-48.

24. Douglas KM, Pace AV, Treharne GJ, et al. Excess recurrent cardiac events in rheumatoid arthritis patients with acute coronary syndrome. Ann Rheum Dis 2006;65:348-53.

25. Ehrlich-Jones L, Lee J, Semanik P, et al. Relationship between beliefs, motivation, and worries about physical activity and physical activity participation in persons with rheumatoid arthritis. Arthritis Care Res 2011;63:1700-5.

26. de Jong $Z$, Munneke $M$, Zwinderman $A H$, et al. Is a long-term high-intensity exercise program effective and safe in patients with rheumatoid arthritis? Results of a randomized controlled trial. Arthritis Rheum 2003;48:2415-24.

27. Plasqui G. The role of physical activity in rheumatoid arthritis. Physiol Behav 2008:94:270-5.

28. Metsios GS, Stavropoulos-Kalinoglou A, Sandoo A, et al. Vascular function and inflammation in rheumatoid arthritis: the role of physical activity. Open Cardiovasc Med J 2010;4:89-96.

29. Stenström $\mathrm{CH}$, Minor MA. Evidence for the benefit of aerobic and strengthening exercise in rheumatoid arthritis. Arthritis Rheum 2003;49:428-34.

30. Robustillo Villarino M, Rodilla Sala E, Gil Latorre F, et al. AB0372 Physical Exercise has no Influence on Markers of Subclinical Cardiovascular Disease in Patients with Rheumatoid Arthritis. Ann Rheum Dis 2015;74(Suppl 2):1018.1-1018.
31. Stavropoulos-Kalinoglou A, Metsios GS, Veldhuijzen van Zanten $\mathrm{JJ}$, et al. Individualised aerobic and resistance exercise training improves cardiorespiratory fitness and reduces cardiovascular risk in patients with rheumatoid arthritis. Ann Rheum Dis 2013;72:1819-25.

32. Cersit HP, Yagci I, Cersit S, et al. Sat0112 the effect of cardiac rehabilitation program on disease activity, functional outcome and cardiovascular risk in patients with rheumatoid arthritis. Ann Rheum Dis 2016;75(Suppl 2):705-6.

33. Shaw I, Shaw BS, Brown GA, et al. Concurrent resistance and aerobic training as protection against heart disease. Cardiovasc $J$ Afr 2010;21:196-9.

34. Committee IR. Guidelines for data processing and analysis of the International Physical Activity Questionnaire (IPAQ)-short and long forms, 2005

35. Medicine ACoS. ACSM's guidelines for exercise testing and prescription. USA: Lippincott Williams \& Wilkins, 2013.

36. Dickens C, McGowan L, Clark-Carter D, et al. Depression in rheumatoid arthritis: a systematic review of the literature with metaanalysis. Psychosom Med 2002;64:52-60.

37. Raison CL, Capuron L, Miller AH. Cytokines sing the blues: inflammation and the pathogenesis of depression. Trends Immunol 2006;27:24-31.

38. Wilson PW, D'Agostino RB, Levy D, et al. Prediction of coronary heart disease using risk factor categories. Circulation 1998;97:1837-47.

39. Anderson TJ, Grégoire J, Hegele RA, et al. 2012 update of the canadian cardiovascular society guidelines for the diagnosis and treatment of dyslipidemia for the prevention of cardiovascular disease in the adult. Can J Cardiol 2013;29:151-67.

40. Bard RL, Rubenfire M, Eagle K, et al. Utility of C-reactive protein measurement in risk stratification during primary cardiovascular disease prevention. Am J Cardiol 2005;95:1378-9.

41. Felson DT, Anderson JJ, Boers M, et al. The American college of rheumatology preliminary core set of disease activity measures for rheumatoid arthritis clinical trials. Arthritis \& Rheumatism 1993;36:729-40.

42. Bruce RA, Kusumi F, Hosmer D. Maximal oxygen intake and nomographic assessment of functional aerobic impairment in cardiovascular disease. Am Heart J 1973;85:546-62.

43. Lerman J, Bruce RA, Sivarajan E, et al. Low-level dynamic exercises for earlier cardiac rehabilitation: aerobic and hemodynamic responses. Arch Phys Med Rehabil 1976;57:355-60.

44. Gibbons RJ, Balady GJ, Bricker JT, et al. Acc/aha 2002 guideline update for exercise testing: Summary article. A report of the american college of cardiology/american heart association task force on practice guidelines (committee to update the 1997 exercise testing guidelines). J Am Coll Cardiol 2002;40:1531-40.

45. Foster C, Jackson AS, Pollock ML, et al. Generalized equations for predicting functional capacity from treadmill performance. Am Heart $J$ 1984:107:1229-34

46. Pollock ML, Foster C, Schmidt D, et al. Comparative analysis of physiologic responses to three different maximal graded exercise test protocols in healthy women. Am Heart J 1982;103:363-73.

47. Craig CL, Marshall AL, Sjöström M, et al. International physical activity questionnaire: 12 -country reliability and validity. Med Sci Sports Exerc 2003;35:1381-95.

48. Metsios GS, Stavropoulos-Kalinoglou A, Treharne GJ, et al. Disease activity and low physical activity associate with number of hospital admissions and length of hospitalisation in patients with rheumatoid arthritis. Arthritis Res Ther 2011;13:R108.

49. Demmelmaier I, Bergman P, Nordgren B, et al. Current and maintained health-enhancing physical activity in rheumatoid arthritis: a cross-sectional study. Arthritis Care Res 2013;65:1166-76.

50. Hernández-Hernández V, Ferraz-Amaro I, Díaz-González F. Influence of disease activity on the physical activity of rheumatoid arthritis patients. Rheumatology 2014;53:722-31.

51. Fries JF, Spitz P, Kraines RG, et al. Measurement of patient outcome in arthritis. Arthritis Rheum 1980;23:137-45.

52. Bruce B, Fries JF. The stanford health assessment questionnaire: Dimensions and practical applications. Health Qual Life Outcomes 2003;1:20.

53. Bruce B, Fries JF. The Health Assessment Questionnaire (HAQ). Clin Exp Rheumatol 2005;23:S14. 